\title{
Dr. Angelika Niebler, MEP
}

\section{Parlamentarische Geschäftsführerin der CDU/CSU-Gruppe im Europäischen Parlament}

\author{
Juristinnen machen Karriere - wir stellen sie vor
}

Das Interview führte die djb-Vizepräsidentin Margarete Hofmann am 11. Juli 2012 in Brüssel.

Fragen an die Politikerin Niebler und zur Politik im europäischen Kontext:

Wie sind Sie zur Politik gekommen? Wann haben Sie sich entschieden, Politikerin zu werden?

Ich bin über mein kommunales Engagement zur Politik gekommen. In meinem Landkreis war ich sehr aktiv und habe zum Beispiel ein elektronisches Bürgernetz aufgebaut. Meine politische Karriere habe ich aber nicht gezielt geplant. Ich bin meiner Partei aufgefallen. Sie haben mich gefragt, ob ich mich hauptamtlich engagieren möchte und für das Europäische Parlament kandidiere. Ich habe allerdings nicht gleich ja gesagt, denn ich war damals schwanger und arbeitete in einer großen Sozietät. Die Stimmung in den Kreisverbänden war aber so positiv, dass ich dann zugesagt habe.

Haben Sie ein Vorbild in der Politik? Sind Vorbilder wichtig für die persönliche Entwicklung?

Ja, Vorbilder sind sehr wichtig. In der Politik war das für mich der damalige CSU-Vorsitzende Edmund Stoiber, der mich motiviert hat, in die CSU einzutreten. Edmund Stoiber ist ein leidenschaftlicher Politiker, er wollte immer Dinge bewegen, den Freistaat Bayern nach vorne bringen. Das hat mich sehr beeindruckt.

Was war bislang Ihr größter politischer Erfolg, was Ihre größte politische Niederlage?

Parteipolitisch war für mich als Vorsitzende der CSU-Frauenunion der größte Erfolg, dass wir das Thema Frauen in der Partei engagiert angegangen sind und die Quote in den oberen Parteigremien durchsetzen konnten. Die spannendste Zeit im Europäischen Parlament war meine Zeit als Ausschussvorsitzende und die erfolgreiche Beratung des Klimapakets (v.a. Richtlinie erneuerbare Energien, Emissionshandel, $\mathrm{CO}_{2}$-Emissionen für PKWs), ebenso die Roaming-Verordnung 2007.

Eine politische Niederlage, die wirklich schmerzhaft war, habe ich zum Glück nicht erlebt. Ich bin der Meinung, wenn etwas nicht im ersten Anlauf klappt, dann muss man es nochmals probieren.

Im Europäischen Parlament (EP) arbeiten Sie mit Kolleginnen und Kollegen aus 27 Mitgliedstaaten zusammen. Welche Rolle spielen dabei Parteigrenzen, welche Rolle spielen nationale Besonderheiten?

Es gibt da keine festgefahrenen Muster. Es hängt ganz vom Thema oder Dossier ab, mit wem man zusammenarbeitet.
Dr. Angelika Niebler, geboren am 18. Februar 1963 in München, studierte zunächst Rechtswissenschaften an der Ludwig Maximilians Universität München und der Universität Genf. 1988 legte sie das 1. Juristische Staatsexamen ab und absolvierte von 1988 bis $1991 \mathrm{ihr}$ Referendariat im Bezirk des Oberlandesgerichts München, im Regierungsbezirk Oberbayern und in einer Anwaltskanzlei in London. 1992, nach erfolgreichem Abschluss des 2. Juristischen Staatsexamens, nahm Angelika Niebler als Stipendiatin des British Councils am European Young Lawyers Course an der Universität Edinburgh teil. Im gleichen Jahr promovierte sie zur Dr. jur. und war von 1991 bis 1999 als Rechtsanwältin, zuletzt als Partnerin, in verschiedenen internationalen Kanzleien in München tätig.

Seit 1995 ist sie Mitglied des Bezirkvorstandes des CSU-Bezirksverbandes Oberbayern, seit 2009 Mitglied im CSU-Parteivorstand und Präsidium. Von 1998 bis 2008 war sie Vorsitzende der FrauenUnion Oberbayern, seit 2009 leitet sie den Landesverband der Frauen-Union Bayern, dem 26.00o Frauen angehören.

Am 20. Juli 1999 wurde Angelika Niebler Mitglied des Europäischen Parlaments. Seit dieser Zeit ist sie parlamentarische Geschäftsführerin der CDU/CSU Gruppe im EP. Sie bekleidete im Parlament verschiedene Funktionen, war unter anderem von 2007 bis zu den Wahlen Mitte 2009 Vorsitzende des Ausschusses für Industrie, Forschung und Energie. Seit 2009 ist sie Präsidentin der Delegation des Europäischen Parlamentes für die Beziehungen zur Arabischen Halbinsel, und sowohl Mitglied im Ausschuss für Industrie, Forschung und Energie und im Ausschuss für die Rechte der Frau und die Gleichstellung der Geschlechter, als auch stellvertretendes Mitglied im Rechtsausschuss.

Angelika Niebler lebt mit ihrem Mann und ihren zwei Söhnen in Vaterstetten. (AB)

Ich will immer die Interessen Deutschlands und Bayerns gut vertreten. Ich versuche dann die jeweiligen Kolleginnen und Kollegen zu gewinnen, die mir helfen können, meine Ziele zu erreichen.

In den Führungsgremien des EP selbst sind weibliche Abgeordnete stark unterrepräsentiert: so sind z. B. von 14 Vizepräsidenten nur drei Frauen. Wie ist es dazu gekommen und wollen Sie hier Abhilfe schaffen?

Die Analyse ist zutreffend, es gibt weniger Frauen in Führungspositionen im Parlament und in den Fraktionen als noch vor ein paar Jahren. Ich glaube, es liegt an unseren Strukturen, viele Funktionen werden noch nach nationalen Vorgaben vergeben, und da haben oft die Männer die Nase vorn. Es ist also 
noch viel zu tun. Je mehr Frauen ins Europäische Parlament gewählt werden, desto leichter wird es.

Was sind die hauptsächlichen Sorgen und Nöte Ihrer Wählerinnen und Wähler, wenn Sie im Wahlkreis Ihre Sprechstunde abhalten?

Das politisch zentrale Thema ist eindeutig die Euro-Krise. Wie halten wir den Euro stabil? Das Thema schlägt seit Monaten alle anderen Themen. Ein weiteres Thema, das die Bürger beschäftigt: Wie schaffen wir es, dass unsere Energiepreise bezahlbar bleiben. katoren und Europa-Botschafter vor Ort. Aber die kommunale Ebene allein reicht nicht. Auch im Bundestag müssen wir Europa erklären!

2014 stehen die nächsten Europawahlen an. Was muss aus Ihrer Sicht getan werden, damit die Bürgerinnen und Bürger das EP stärker als das demokratische Organ der Vertretung der europäischen Bürger wahrnehmen und zu den Wahlurnen gehen?

Nochmals, das Werben für Europa ist ein permanenter Prozess. Ich versuche jede freie Minute für die Idee Europa zu

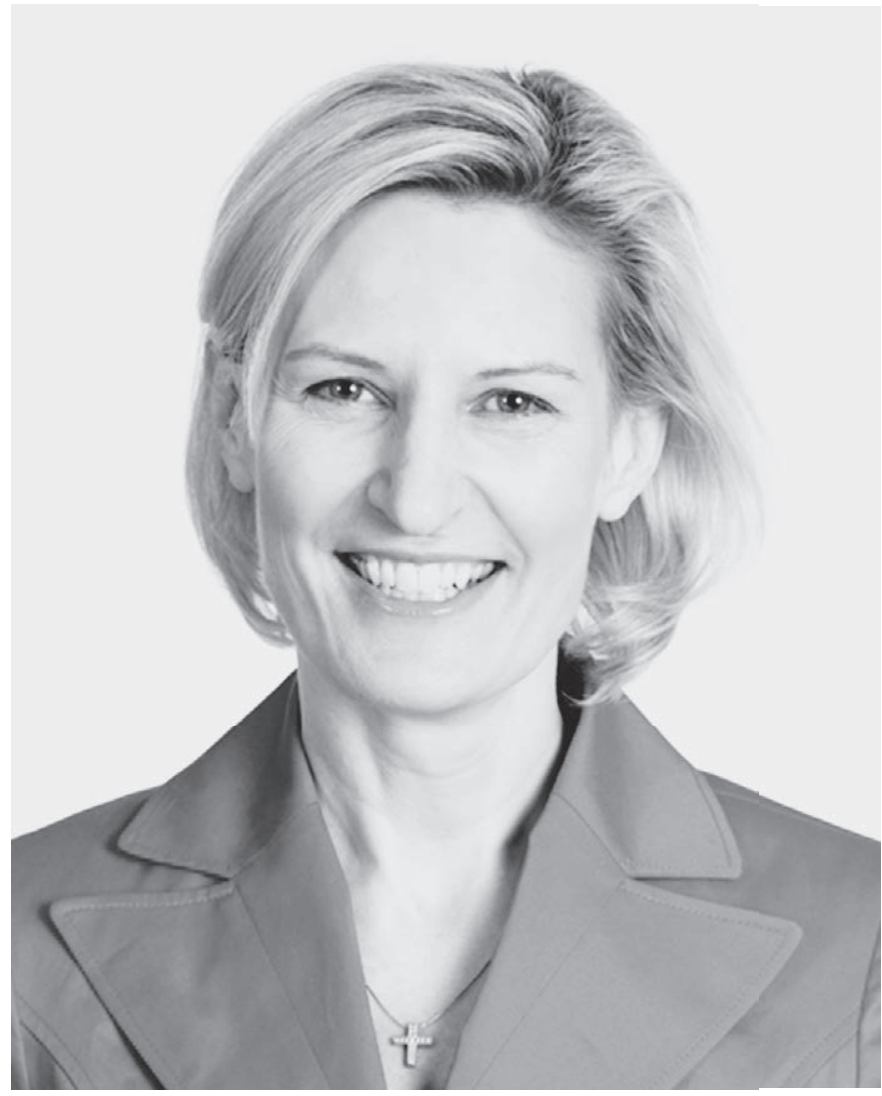

Die Bürgerinnen und Bürger haben heute mehr denn je den Eindruck, dass angesichts der ökonomischen und finanziellen Krise in Europa wichtige Entscheidungen „über ihren Kopf" hinweg getroffen werden.

Auf Ihrer Website bezeichnen Sie sich selbst als „Ihre Brücke nach Europa“. Wie können wir wieder das Vertrauen der Bürgerinnen und Bürger in Europa herstellen - nicht nur in Deutschland, wo wir uns vor allem als Zahlmeister verstehen, sondern auch in den krisengeschüttelten südlichen Mitgliedstaaten?

Wir dürfen nicht zulassen, dass Europa und die EU immer wieder zum Sündenbock abgestempelt werden. Stattdessen müssen wir erklären, dass wir für bestimmte Themen und Probleme ein starkes Europa brauchen. Ich halte es deshalb für ganz wichtig, so viel wie möglich vor Ort bei den Bürgern zu sein und für Europa einzutreten. Wir brauchen mehr Multipli- werben und bin nicht nur in Brüssel, sondern auch in meinem Wahlkreis viel unterwegs. Die Brücke nach Europa verstehe ich sehr wörtlich. Ich hoffe natürlich, dass mein Engagement mit Blick auf die Europawahlen 2014 honoriert wird.

Ein weiterer Aspekt: Das Europäische Parlament ist so transparent wie kein anderes Parlament in der Welt. Alle Dokumente des Plenums und der Ausschüsse sind online verfügbar. Die Bürger können also leicht alle gewünschten Dokumente abrufen.

\section{Fragen zur Frauen- und Gleichstellungspolitik:}

Sie haben als Vorsitzende der Frauenunion in der CSU eine Quote von 40 Prozent für die Besetzung der oberen Parteigremien durchgesetzt. Für Ihre doch noch recht traditionell geprägte Partei muss das eine kleine Revolution gewesen 
sein. Wie haben Sie diese Revolution durchgesetzt? Was bzw. wer hat Ihnen dabei geholfen?

Persönlich habe ich viel Überzeugungsarbeit in die Partei hinein geleistet und unzählige Gespräche geführt. Es gab glücklicherweise auch viele engagierte Mitstreiter und Mitstreiterinnen, allen voran der Parteivorsitzende Horst Seehofer. Dennoch ist es ziemlich knapp ausgegangen: 53 Prozent zu 47 Prozent. Aber Mehrheit ist Mehrheit!

Finden sich denn genügend jüngere Frauen, die in der Partei auch Verantwortung übernehmen wollen? Was tun Sie, damit Frauen sich engagieren und sich auch auf höhere Posten bewerben?

Die Frauen-Union macht hier sehr viel. So gibt es zum Beispiel ein Mentoring-Programm, das vor einigen Jahren ins Leben gerufen wurde. Aber das allein reicht nicht. Sie müssen Frauen gezielt ansprechen, um sie für die Politik zu gewinnen. Wir tun viel, um sie zu professionalisieren. Wir müssen Mut machen, damit die Frauen sich auch um Funktionen und Ämter bewerben, und vermitteln, dass Politik auch Freude macht und zu einer hohen Zufriedenheit führen kann.

Bei Führungspositionen in der Wirtschaft befürworten Sie eine verbindliche Quote für den Aufsichtsrat, allerdings nicht für den Vorstand. Warum meinen Sie, reicht das aus, um den Frauenanteil in allen wirtschaftlichen Führungspositionen denn dies sollte das Ziel sein - entscheidend zu erhöhen? Ich glaube an eine Hebelwirkung, denn der Aufsichtsrat beruft den Vorstand. Wenn wir mehr Frauen im Aufsichtsrat haben, wird der Blick auf qualifizierte Frauen ein anderer sein, man wird zielgerichteter auf sie zugehen. Bei Unternehmen widerstrebt es mir aus Prinzip, in das operative Geschäft dirigistisch einzugreifen.

Die Quotendebatte dominiert derzeit die öffentliche Diskussion. Welche anderen für Frauen relevanten Themen sollten aus Ihrer Sicht vorrangig angegangen werden?

Drei Themen, die nicht neu sind, aber immer noch aktuell: Altersarmut von Frauen, Lohndifferenz und Gewalt gegen Frauen.

Eine derzeit unvermeidliche Frage: Was halten Sie persönlich vom (Streit um's) Betreuungsgeld?

Ich finde, wir sollten hier keinen Glaubenskrieg führen. Ich bin für das Betreuungsgeld, weil es wichtig ist, dass wir einen Ausdruck der Wertschätzung für Familien finden, für das, was im privaten Umfeld für Kinder geleistet wird. Meine Grundüberzeugung ist, dass starke Familienstrukturen für die Weiterentwicklung der Gesellschaft eine wesentliche Rolle spielen. Alles, was Familien stärkt, unterstütze ich.

Persönliche Fragen (und eine letzte Frage zum djb):

Wie sind Sie zum Jurastudium gekommen? Was gefällt Ihnen besonders an der Juristerei?
Ich habe bei einer Anwaltskanzlei als Schülerin ein Praktikum gemacht, das hat mich motiviert, Jura zu studieren. Ich vertrete gern Interessen und arbeite gern an einem Interessenausgleich. Insofern passt das auch zur Politik, wie wir sie hier machen.

Sie haben direkt nach dem 2. Staatsexamen mehrere Jahre als Anwältin in international ausgerichteten Großkanzleien gearbeitet. Wie sehr hat Sie diese berufliche Etappe geprägt?

Ich blicke noch heute mit großer Begeisterung auf meine Anwaltstätigkeit zurück, ich habe das mit Leidenschaft gemacht, zuletzt als Partnerin. Ich war mir zunächst nicht sicher, ob mir Politik genauso gut gefällt wie meine Anwaltstätigkeit. Es gibt hier aber viele Parallelen. Ich habe den Arbeitsstil in der Sozietät mit ins Europäische Parlament genommen. Akquise betreiben, unkonventionell denken, konstruktive Lösungen suchen und finden, das gehört zu Anwaltschaft und Politik gleichermaßen, genauso wie die Leidenschaft, der volle Einsatz und das harte Arbeiten weit über die 40-Stundenwoche hinaus.

Sie haben zwei Söhne im Alter von 13 und fünf Jahren. Ihren zweiten Sohn haben Sie bekommen, als Sie Vorsitzende des mächtigen Industrieausschusses waren. Wie vereinbaren Sie ganz konkret Beruf und Familie?

Ich habe viele helfende Hände, allen voran seit über 13 Jahren eine gelernte Kinderpflegerin im Haus. Dann braucht man eine gute Organisation, hohe Flexibilität und viel Gelassenheit. Und ich habe mir den richtigen Mann ausgesucht, der mein politisches Engagement auch voll mit unterstützt.

Wie erholen Sie sich von der Parlamentsarbeit? Können Sie überhaupt abschalten mit Ihrem Fulltime-Job, der ja auch vor dem Wochenende nicht Halt macht?

Abschalten kann ich sehr gut, am besten mit den Kindern, die einen sofort wieder auf den Boden der Realität zurückholen. Viel Bewegung an der frischen Luft ist für mich auch ganz wichtig. Leider bleibt für persönliche Hobbies kaum Zeit.

Was würden Sie gerne einmal machen, wenn Sie mehr freie Zeit hätten?

Ich würde sehr gerne mehr am kulturellen Leben teilnehmen und zum Beispiel ins Theater gehen. Außerdem einfach mein Land und die Landschaft genießen, draußen unterwegs sein, wandern, Fahrrad fahren. Man macht mich nicht glücklich, wenn man mir eine Weltreise anbietet. Ich bin glücklich in der vertrauten Umgebung zu Hause, wenn ich keine feste Agenda habe und spontan etwas machen kann. Ja, und ich hätte gern wieder mehr Zeit für meine Freunde.

Sie verbringen nun schon 13 Jahre mehrere Tage in der Woche in Brüssel. Bekommen Sie etwas vom Leben in der Stadt mit? Was schätzen Sie an Brüssel, was gefällt Ihnen weniger? 
Ich schätze an Brüssel die gute Küche. Was ich überhaupt nicht schätze, ist die hohe Kriminalität. [N.B.: MEP Niebler wurde 2010 Opfer eines Überfalls.]

Politiker/in sein ist ein Amt auf Zeit. Können Sie sich ein Leben nach der Politik vorstellen? Was würden Sie dann am liebsten tun?

Natürlich, gar kein Problem. Ich würde sofort wieder in meinen alten Beruf zurückgehen, den ich mit Herzblut ausgefüllt habe. Ich müsste mich nicht neu erfinden.
Sie sind seit langen Jahren djb-Mitglied. Mit welchen Argumenten würden Sie junge Juristinnen überzeugen, in den djb einzutreten?

Zwei Punkte möchte ich nennen: der djb ist eine starke Interessenvertretung und hat ein hervorragendes Netzwerk. Ohne ein gutes Netzwerk geht es weder in der Politik noch in der Wirtschaft oder im Berufsleben.

\section{Frau Niebler, ich danke Ihnen für dieses Gespräch.}

\section{Impressum}

Schriftleitung

Anke Gimbal, Rechtsassessorin (V.i.S.d.P.)

Juliane Lindner

\section{Redaktionsanschrift}

Deutscher Juristinnenbund e. V.

Anklamer Str. 38

10115 Berlin

Telefon: 030 443270-0

Telefax: 030 443270-22

E-Mail: geschaeftsstelle@djb.de

www.djb.de

\section{Erscheinungsweise:}

4 Ausgaben im Jahr

\section{Bezugspreise 2012}

Jahresabonnement 52,- $€$; Einzelheft 14,- $€$.

Alle Preise verstehen sich incl. MWSt, zzgl. Vertriebskosten.

Bestellmöglichkeit

Bestellungen beim örtlichen Buchhandel oder direkt bei der Nomos Verlagsgesellschaft Baden-Baden

\section{Kündigungsfrist}

jeweils drei Monate vor Kalenderjahresende
Bankverbindung generell

Zahlungen jeweils im Voraus an Nomos Verlagsgesellschaft, Postbank Karlsruhe: BLZ 660 100 75, Konto Nr. 73636-751 oder Sparkasse Baden-Baden Gaggenau: BLZ 662500 30, Konto Nr. 5-002266

\section{Druck und Verlag}

Nomos Verlagsgesellschaft $\mathrm{mbH} \&$ Co. KG

Waldseestr. 3-5, D-76530 Baden-Baden

Telefon (07221) 2104-O/Fax (07221) 2104-27

E-Mailnomos@nomos.de

\section{Anzeigen}

Sales friendly Verlagsdienstleistungen

Siegburger Str. 123, 53229 Bonn

Telefon (0228) 978980, Fax (o228) 9789820

E-Mail roos@sales-friendly.de

Urheber- und Verlagsrechte

Die Zeitschrift sowie alle in ihr enthaltenen einzelnen Beiträge und Abbildungen sind urheberrechtlich geschützt. Jede Verwertung, die nicht ausdrücklich vom Urheberrechtsgesetz zugelassen ist, bedarf der vorherigen Zustimmung des Verlags.

Mit der Annahme zur Veröffentlichung überträgt der Autor dem Verlag das ausschließliche Verlagsrecht für die Zeit bis zum Ablauf des Ur- heberrechts. Eingeschlossen sind insbesondere auch das Recht zur Herstellung elektronischer Versionen und zur Einspeicherung in Datenbanken sowie das Recht zu deren Vervielfältigung und Verbreitung online oder offline ohne zusätzliche Vergütung. Nach Ablauf eines Jahres kann der Autor anderen Verlagen eine einfache Abdruckgenehmigung erteilen; das Recht an der elektronischen Version verbleibt beim Verlag.

Namentlich gekennzeichnete Beiträge geben nicht in jedem Fall die Meinung der Herausgeber/Redaktion oder des Verlages wieder. Unverlangt eingesendete Manuskripte - für die keine Haftung übernommen wird - gelten als Veröffentlichungsvorschlag zu den Bedingungen des Verlages.

Die Redaktion behält sich eine längere Prüfungsfrist vor. Eine Haftung bei Beschädigung oder Verlust wird nicht übernommen. Bei unverlangt zugesandten Rezensionsstücken keine Garantie für Besprechung oder Rückgabe. Es werden nur unveröffentlichte Originalarbeiten angenommen. Die Verfasser erklären sich mit einer nicht sinnentstellenden redaktionellen Bearbeitung einverstanden.

ISSN 1866-377X 